\title{
Complete $\alpha^{7} m$ Lamb shift of helium triplet states
}

\author{
Vojtěch Patkóš, ${ }^{1}$ Vladimir A. Yerokhin, ${ }^{2}$ and Krzysztof Pachucki ${ }^{3}$ \\ ${ }^{1}$ Faculty of Mathematics and Physics, Charles University, \\ Ke Karlovu 3, 12116 Prague 2, Czech Republic \\ ${ }^{2}$ Center for Advanced Studies, Peter the Great St. Petersburg Polytechnic University, \\ Polytekhnicheskaya 29, 195251 St. Petersburg, Russia \\ ${ }^{3}$ Faculty of Physics, University of Warsaw, Pasteura 5, 02-093 Warsaw, Poland
}

(Dated: April 8, 2021)

\begin{abstract}
We have derived the complete formula for the $\alpha^{7} m$ contribution to energy levels of an arbitrary triplet state of the helium atom, performed numerical calculations for the $2^{3} S$ and $2^{3} P$ states, and thus improved the theoretical accuracy of ionization energies of these states by more than an order of magnitude. Using the nuclear charge radius extracted from the muonic helium Lamb shift, we obtain the theoretical prediction in excellent agreement with the measured $2^{3} S-2^{3} P$ transition energy [X. Zheng et al., Phys. Rev. Lett. 199, 263002 (2017)]. At the same time we observe significant discrepancies with experiments for the $2^{3} S-3^{3} D$ and $2^{3} P-3^{3} D$ transitions.
\end{abstract}

\section{INTRODUCTION}

High precision spectroscopic measurements in atoms and molecules can be used for the determination of fundamental constants such as the Rydberg constant [1] and the electron-nuclear mass ratio $[2,3]$. They can also be used for the determination of nuclear properties, among them magnetic dipole and electric quadrupole moments. In the present work we investigate the possibility of determining the nuclear charge radius by means of atomic spectroscopy.

The differences of the (squares of) nuclear charge radii between different isotopes are routinely determined nowadays from measurements of the isotope shifts of transition frequencies [4-7]. Here we address a more ambitious task of determining the absolute value of the nuclear charge radius, specifically, that of the helium atom. The main motivation of the spectroscopic determination of nuclear radii is to make possible a comparison of different methods, such as electron scattering and the muonicatom spectroscopy, and to search for possible deviations that might signal the existence of unknown interactions at the atomic scale.

The spectroscopic determination of the nuclear radius has already been accomplished for the hydrogen atom. Importantly, it was performed by two independent methods: from ordinary hydrogen [8-11] and from muonic hydrogen $[12,13]$. At first the comparison of the two methods revealed a large discrepancy, which became known as the proton size puzzle. This discrepancy seems to be close to a resolution now [14] because several recent spectroscopic and scattering experiments showed to be consistent with the muonic hydrogen proton radius. As a result, the comparison of ordinary and muonic hydrogen has provided improved values for the proton radius and the Rydberg constant and forced a reconsideration of systematic effects in hydrogen spectroscopy.

One may expect that a similar comparison performed for other nuclei will also reveal interesting findings. An important step towards such a comparison is the recent muonic helium experiment [15], which determined the charge radius of the helium- 4 nucleus (the $\alpha$ particle) with a $0.05 \%$ precision.

The goal of the present work is to improve the theoretical accuracy of the $2^{3} S-2^{3} P$ transition energy in atomic helium to a level sufficient for the determination of the nuclear charge radius from the existing measurements in ordinary helium. We achieve this by performing the complete calculation of the $\alpha^{7} m$ QED effects. Unfortunately, we also find that our calculation does not resolve the previously reported discrepancy of theoretical predictions with experimental results for the $2^{3} S-2^{3} D$ and $2^{3} P-2^{3} D$ transitions [16]. In view of this, we postpone the determination of the $\alpha$-particle charge radius until these discrepancies are resolved. Henceforth, we present our calculations of the complete $\alpha^{7} m$ QED effects and obtain the improved theoretical predictions for atomic helium energy levels using the quantum electrodynamic theory.

\section{PERTURBATIVE EXPANSION OF ATOMIC ENERGY LEVELS}

The basic assumption in bound-state quantum electrodynamics is the possibility of the expansion of the bound state energy $E$ in a power series of the fine-structure constant $\alpha$,

$$
\begin{aligned}
E\left(\alpha, \frac{m}{M}\right)= & \alpha^{2} E^{(2)}\left(\frac{m}{M}\right)+\alpha^{4} E^{(4)}\left(\frac{m}{M}\right)+\alpha^{5} E^{(5)}\left(\frac{m}{M}\right) \\
& +\alpha^{6} E^{(6)}\left(\frac{m}{M}\right)+\alpha^{7} E^{(7)}\left(\frac{m}{M}\right)+\ldots
\end{aligned}
$$

where $m / M$ is the electron-to-nucleus mass ratio and the expansion coefficients $E^{(n)}$ may contain finite powers of $\ln \alpha$. The coefficients $E^{(i)}(m / M)$ are further expanded in powers of the $m / M$ ratio,

$$
E^{(i)}\left(\frac{m}{M}\right)=E^{(i, 0)}+\frac{m}{M} E^{(i, 1)}+\left(\frac{m}{M}\right)^{2} E^{(i, 2)}+\ldots
$$


The leading expansion term $E_{0} \equiv E^{(2,0)}$ is the nonrelativistic energy, which is the eigenvalue of the nonrelativistic Hamiltonian $H_{0}$. For the helium atom,

$$
H_{0}=\frac{p_{1}^{2}}{2}+\frac{p_{2}^{2}}{2}-\frac{Z}{r_{1}}-\frac{Z}{r_{2}}+\frac{1}{r},
$$

where $r=\left|\vec{r}_{1}-\vec{r}_{2}\right|$. Further expansion terms in Eqs. (1) and (2) can be expressed as expectation values of some effective Hamiltonians with the nonrelativistic wave function. The derivation of the effective Hamiltonians is the central problem, and this can be accomplished within the approach of the nonrelativistic QED (NRQED), which is employed here. While the leading-order terms are simple, the derivation becomes increasingly complicated for high powers of $\alpha$. The complete theory of helium energy levels up to order $\alpha^{6} m$ was reviewed in our former work [17]. In the present work, we summarize the $\alpha^{7} m$ contribution and perform its numerical calculations for the $2^{3} S$ and $2^{3} P$ states. In this calculation we assume the infinitely heavy nucleus. The corresponding finite nuclear mass corrections are much smaller than the uncertainty due to the approximate calculation of the next order $\alpha^{8} m$ contribution, and therefore they are neglected.

\section{III. $\alpha^{7} m$ CONTRIBUTION}

The $\alpha^{7} m$ contribution $E^{(7)}$ is represented as a sum of three terms,

$$
E^{(7)}=E_{L}^{(7)}+E_{\text {exch }}^{(7)}+E_{\mathrm{rad}}^{(7)}
$$

where $E_{L}^{(7)}$ is the low-energy part - specifically, the relativistic correction to the so-called Bethe logarithm; $E_{\text {exch }}^{(7)}$ is the part induced by the electron-electron and electronnucleus photon exchange; and $E_{\text {rad }}^{(7)}$ is induced by the radiative QED effects beyond those accounted for by $E_{L}^{(7)}$. Both $E_{\text {exch }}^{(7)}$ and $E_{\text {rad }}^{(7)}$ have the same general structure, being the sum of the first-order and second-order perturbation corrections,

$E_{\mathrm{exch} / \mathrm{rad}}^{(7)}=\left\langle H_{\mathrm{exch} / \mathrm{rad}}^{(7)}\right\rangle+2\left\langle H^{(4)} \frac{1}{\left(E_{0}-H_{0}\right)^{\prime}} H_{\mathrm{exch} / \mathrm{rad}}^{(5)}\right\rangle$.

Here, $H^{(4)}$ is the leading relativistic Breit Hamiltonian (see Eq. (7) of Ref. [19]) and $H^{(5)}$ is the QED $\alpha^{5} m$ Hamiltonian.

The relativistic correction to the Bethe logarithm was derived and calculated numerically in Ref. [18], the photon-exchange contribution was derived in Ref. [19], and the radiative contribution was recently derived in Ref. [20].

\section{A. Relativistic correction to the Bethe logarithm}

We start with the low-energy part in the leading QED contribution. The leading nonrelativistic (dipole) low-
TABLE I. Relativistic corrections to the Bethe logarithm for the $2^{3} S$ and $2^{3} P$ (centroid) states of helium, in units of $\alpha^{7} \mathrm{~m}$.

\begin{tabular}{lcc}
\hline \hline Term & $2^{3} S$ & $2^{3} P$ \\
\hline$E_{L 1}$ & $-45.1291(35)$ & $-41.7175(40)$ \\
$E_{L 2}$ & $335.8675(36)$ & $319.1601(36)$ \\
$E_{L 3}$ & $-1095.0439(3)$ & $-1045.271(8)$ \\
\hline \hline
\end{tabular}

energy contribution of order $\alpha^{5} m$ is given by

$$
\begin{aligned}
E_{L 0}(\Lambda)= & e^{2} \int_{k<\Lambda} \frac{d^{3} k}{(2 \pi)^{3} 2 k}\left(\delta^{i j}-\frac{k^{i} k^{j}}{k^{2}}\right) \\
& \times\left\langle P^{i} \frac{1}{E_{0}-H_{0}-k} P^{j}\right\rangle,
\end{aligned}
$$

where $\vec{P}=\vec{p}_{1}+\vec{p}_{2}$ and $\Lambda=\lambda \alpha^{2}$ is the high-momentum cutoff. $E_{L 0}(\Lambda)$ diverges when $\lambda \rightarrow \infty$, due to the presence of terms proportional to $\lambda$ and $\ln \lambda$. We obtain the finite part of $E_{L 0}$ by subtracting all these $\lambda$ dependent terms. The result is by definition the low-energy $m \alpha^{5}$ contribution, also known as the Bethe logarithm.

The relativistic correction to the Bethe logarithm, $E_{L}^{(7)}$, is obtained similarly. It consists of three parts,

$$
E_{L}^{(7)}=E_{L 1}+E_{L 2}+E_{L 3} .
$$

The first part $E_{L 1}$ is a perturbation of the nonrelativistic low-energy contribution $E_{L 0}$ in Eq. (6) by the Breit Hamiltonian $H^{(4)}$, the second part $E_{L 2}$ is induced by the relativistic correction to the current operator $\vec{P} / m$, and the third term $E_{L 3}$ is the retardation correction. All of these corrections are defined as remainders after dropping $\lambda$-divergent terms $\sim \lambda^{2}, \lambda, \ln \lambda$, and $\ln ^{2} \lambda$. The divergent terms are cancelled when combined with corresponding terms from the other contributions in Eq. (4).

Numerical results for $E_{L 1}, E_{L 2}$ and $E_{L 3}$ are taken from Ref. [18] and are summarized in Table I. Numerical uncertainties are negligible in comparison to uncertainties due to higher order corrections.

\section{B. Photon-exchange contribution}

The contribution $E_{\text {exch }}^{(7)}$ is induced by the electronelectron and electron-nucleus photon exchanges, i.e., in its definition we exclude all diagrams with photons emitted and absorbed by the same electron. We split this contribution into the first-order and second-order parts,

$$
E_{\text {exch }}^{(7)}=\left\langle H_{\text {exch }}^{(7)}\right\rangle+E_{\text {exch }}^{\mathrm{sec}},
$$

where

$$
E_{\mathrm{exch}}^{\mathrm{sec}}=2\left\langle H_{\mathrm{exch}}^{(5)} \frac{1}{\left(E_{0}-H_{0}\right)^{\prime}} H^{(4)}\right\rangle,
$$


and

$$
H_{\mathrm{exch}}^{(5)}=-\frac{7}{6 \pi} \frac{1}{r^{3}}
$$

It is advantageous to express $\left\langle H_{\mathrm{exch}}^{(7)}\right\rangle$ using a set of operators $Q_{i}$ with $i=1 \ldots 64$ which are suited for a numerical evaluation and are summarized in Table IV. The first 50 of these operators were defined in Refs. [21-23], whereas the remaining 14 operators are exclusive for the $\alpha^{7} \mathrm{~m}$ contribution. The final expression for the photon-exchange contribution is

$$
\begin{aligned}
E_{\mathrm{exch}}^{(7)}= & \frac{1}{\pi}\left\{\ln \frac{\alpha^{-2}}{2}\left(-\frac{22}{45} Z Q_{3}-\frac{19}{90} Q_{6 T}-\frac{4}{15} Q_{10}+\frac{2}{15} Z Q_{18}-\frac{1}{15} Z Q_{62}\right)+\left(-\frac{772}{675}+\frac{22}{45} \ln 2\right) Z Q_{3}\right. \\
& +\left(\frac{7937}{2700}-\frac{9}{10} \ln 2\right) Q_{6 T}+\left(-\frac{617}{1800}-\frac{8}{15} \ln 2\right) Q_{10}+\left(\frac{841}{1800}+\frac{2}{3} \ln 2\right) Z Q_{18}+\frac{31}{240} Q_{25}+\frac{44}{45} Z Q_{52} \\
& \left.+\frac{8}{15} Q_{54}-\frac{33}{10} Q_{55}-\frac{4}{15} Z Q_{58}-\frac{7}{10} Q_{60}+\frac{7}{24} Q_{61}+\left(\frac{14}{225}+\frac{1}{15} \ln 2\right) Z Q_{62}+\frac{2}{15} Z Q_{63}\right\}+E_{\mathrm{exch}}^{\mathrm{sec}} .
\end{aligned}
$$

The above expression was obtained by slightly simplifying our former result in Ref. [19] with help of the following expectation value identity

$$
p^{i}\left(\frac{\delta^{i j}}{r^{3}}-3 \frac{r^{i} r^{j}}{r^{5}}\right) p^{j}=\frac{2 \pi}{3} \vec{p} \delta^{3}(r) \vec{p}+\frac{Z}{4}\left(\frac{\vec{r}_{1}}{r_{1}^{3}}-\frac{\vec{r}_{2}}{r_{2}^{3}}\right) \cdot \frac{\vec{r}}{r^{3}}-\frac{1}{2 r^{4}} .
$$

The expression (9) for $E_{\text {exch }}^{\mathrm{sec}}$ is finite but numerically unstable. We thus regularize it as

$$
\begin{aligned}
E_{\mathrm{exch}}^{\mathrm{sec}}= & 2\left\langle H_{\mathrm{exch}}^{(5)} \frac{1}{\left(E_{0}-H_{0}\right)^{\prime}} H_{R}\right\rangle \\
& +\frac{7}{6 \pi}\left[Q_{9}\left(Z Q_{53}-Q_{7}\right)+Q_{10}-Z Q_{59}\right],
\end{aligned}
$$

where the regularized Breit operator $H_{R}$ is acting on ketstate $|\phi\rangle$ as

$$
\begin{aligned}
H_{R}|\phi\rangle= & \left(-\frac{1}{2}\left(E_{0}-V\right)^{2}-\frac{Z}{4} \frac{\vec{r}_{1} \cdot \vec{\nabla}_{1}}{r_{1}^{3}}-\frac{Z}{4} \frac{\vec{r}_{2} \cdot \vec{\nabla}_{2}}{r_{2}^{3}}\right. \\
& \left.+\frac{1}{4} \nabla_{1}^{2} \nabla_{2}^{2}+\nabla_{1}^{i} \frac{1}{2 r}\left(\delta^{i j}+\frac{r^{i} r^{j}}{r^{2}}\right) \nabla_{2}^{j}\right)|\phi\rangle,(14)
\end{aligned}
$$

where $E_{0}$ is the nonrelativistic energy of $\phi$ and $V=-\frac{Z}{r_{1}}-$ $\frac{Z}{r_{2}}+\frac{1}{r} . H_{R}$ is equivalent to $H^{(4)}$ in the sense that their expectation values on $\phi$ are the same.

\section{The radiative contribution}

The radiative correction $E_{\text {rad }}^{(7)}$ consists of the one-loop self-energy, the one-loop vacuum polarization, the twoloop correction, and the three-loop correction,

$$
E_{\mathrm{rad}}^{(7)}=E_{\mathrm{SE}}^{(7)}+E_{\mathrm{VP}}^{(7)}+E_{\mathrm{rad} 2}^{(7)}+E_{\mathrm{rad} 3}^{(7)} .
$$

The one-loop self-energy contribution is

$$
\begin{aligned}
E_{\mathrm{SE}}^{(7)}= & \frac{1}{\pi}\left\{-\frac{20}{9} E_{0} E^{(4)}+\left(\frac{491}{1800} E_{0}+\frac{3641}{3600} Z^{2}-\frac{1289}{360} Z^{2} \ln 2-\frac{5}{36} \pi^{2} Z^{2}+\frac{8}{3} Z^{2} \ln ^{2} 2+\frac{5}{18} Q_{7}+\frac{5}{4} Z^{2} \zeta(3)\right) Z Q_{1}\right. \\
& -\frac{491+509 Z}{1800} Z Q_{3}+\frac{509}{3600} Z Q_{4}-\frac{1039}{1350} Q_{6 T}+\frac{10}{9} E^{(4)} Q_{7}+\left(\frac{403}{90}-\frac{2}{3} \ln 2\right) Q_{10}+\frac{10}{9} E_{0} Z^{2} Q_{11} \\
& +\frac{20}{9} E_{0} Z^{2} Q_{12}-\frac{20}{9} E_{0} Z Q_{13}-\frac{20}{9} Z^{2} Q_{14}+\frac{20}{9} Z^{3} Q_{15}-\frac{10}{9} Z^{2} Q_{16}+\frac{10}{9} Z Q_{17}-\frac{1271}{360} Z Q_{18} \\
& +\frac{5}{9} Z^{2} Q_{21}+\frac{5}{9} Z^{2} Q_{22}+\frac{10}{9} Z Q_{24}+\frac{1}{60} Q_{25}-\frac{5}{9} Z Q_{28}+\frac{779}{3600} Z Q_{51}+\frac{10}{9} E_{0}^{2} Z Q_{53}-\frac{4}{3} Q_{54} \\
& +\frac{163}{120} Z^{2} Q_{57}+\ln \frac{\alpha^{-2}}{2}\left[-\frac{8}{3} E_{0} E^{(4)}+\left(-\frac{E_{0}}{15}+\frac{53}{120} Z^{2}+\frac{5}{3} Z^{2} \ln 2+\frac{1}{3} Q_{7}\right) Z Q_{1}+\frac{1-11 Z}{15} Z Q_{3}\right. \\
& +\frac{11}{30} Z Q_{4}-\frac{49}{45} Q_{6 T}+\frac{4}{3} E^{(4)} Q_{7}+2 Q_{10}+\frac{4}{3} E_{0} Z^{2} Q_{11}+\frac{8}{3} E_{0} Z^{2} Q_{12}-\frac{8}{3} E_{0} Z Q_{13}-\frac{8}{3} Z^{2} Q_{14} \\
& +\frac{8}{3} Z^{3} Q_{15}-\frac{4}{3} Z^{2} Q_{16}+\frac{4}{3} Z Q_{17}-\frac{8}{3} Z Q_{18}+\frac{2}{3} Z^{2} Q_{21}+\frac{2}{3} Z^{2} Q_{22}+\frac{4}{3} Z Q_{24}-\frac{2}{3} Z Q_{28} \\
& \left.\left.+\frac{11}{30} Z Q_{51}+\frac{4}{3} E_{0}^{2} Z Q_{53}+Z^{2} Q_{57}\right]-\frac{1}{2} \ln ^{2} \frac{\alpha^{-2}}{2} Z^{3} Q_{1}\right\}+E_{\mathrm{SE}}^{\mathrm{sec}}
\end{aligned}
$$


where $E^{(4)}=\left\langle H^{(4)}\right\rangle$ is the Breit correction to the energy. The above formula for $E_{\mathrm{SE}}^{(7)}$ is obtained by simplifying our former result in Ref. [20] with help of the identity (12). The second-order part $E_{\mathrm{SE}}^{\mathrm{sec}}$ is

$$
E_{\mathrm{SE}}^{\mathrm{sec}}=2\left\langle H^{\prime \prime(5)} \frac{1}{\left(E_{0}-H_{0}\right)^{\prime}} H^{\prime \prime(4)}\right\rangle+\frac{1}{\pi}\left(\frac{5}{9}+\frac{2}{3} \ln \frac{\alpha^{-2}}{2}\right)\left\langle H_{R}^{\prime} \frac{1}{\left(E_{0}-H_{0}\right)^{\prime}} H_{R}\right\rangle .
$$

Here, the operators $H^{\prime \prime(4)}$ and $H^{\prime \prime(5)}$ are obtained, respectively, as the $\alpha^{4}$ and $\alpha^{5}$ parts of the spin-dependent Breit Hamiltonian with anomalous magnetic moment (see, e.g., Eq. (1) of Ref. [24]),

$$
\begin{aligned}
& H_{\mathrm{fs}}=H^{\prime \prime(4)}+H^{\prime \prime(5)}+O\left(\kappa^{2}\right)=H_{B}+H_{C}+H_{D}, \\
& H_{B}=\left[\frac{Z}{4}\left(\frac{\vec{r}_{1}}{r_{1}^{3}} \times \vec{p}_{1}+\frac{\vec{r}_{2}}{r_{2}^{3}} \times \vec{p}_{2}\right)(1+2 \kappa)-\frac{3}{4} \frac{\vec{r}}{r^{3}} \times\left(\vec{p}_{1}-\vec{p}_{2}\right)\left(1+\frac{4 \kappa}{3}\right)\right] \frac{\vec{\sigma}_{1}+\vec{\sigma}_{2}}{2}=\left(\vec{Q}_{B}+\kappa \vec{Q}_{B}^{\prime}\right) \frac{\vec{\sigma}_{1}+\vec{\sigma}_{2}}{2}, \\
& H_{C}=\left[\frac{Z}{4}\left(\frac{\vec{r}_{1}}{r_{1}^{3}} \times \vec{p}_{1}-\frac{\vec{r}_{2}}{r_{2}^{3}} \times \vec{p}_{2}\right)(1+2 \kappa)+\frac{1}{4} \frac{\vec{r}}{r^{3}} \times\left(\vec{p}_{1}+\vec{p}_{2}\right)\right] \frac{\vec{\sigma}_{1}-\vec{\sigma}_{2}}{2}=\left(\vec{Q}_{C}+\kappa \vec{Q}_{C}^{\prime}\right) \frac{\vec{\sigma}_{1}-\vec{\sigma}_{2}}{2}, \\
& H_{D}=\frac{1}{4}\left(\frac{\vec{\sigma}_{1} \vec{\sigma}_{2}}{r^{3}}-3 \frac{\vec{\sigma}_{1} \cdot \vec{r} \vec{\sigma}_{2} \cdot \vec{r}}{r^{5}}\right)(1+\kappa)^{2}=\left(Q_{D}^{i j}+\kappa Q_{D}^{\prime i j}\right) \frac{1}{2} \sigma_{1}^{i} \sigma_{2}^{j}+O\left(\kappa^{2}\right),
\end{aligned}
$$

where $\kappa=\alpha / 2 \pi$ is anomalous magnetic moment correction. $H_{R}$ is defined in Eq. (14), and $H_{R}^{\prime}$ is

$$
H_{R}^{\prime}|\phi\rangle=-2 Z\left(\frac{\vec{r}_{1} \cdot \vec{\nabla}_{1}}{r_{1}^{3}}+\frac{\vec{r}_{2} \cdot \vec{\nabla}_{2}}{r_{2}^{3}}\right)|\phi\rangle
$$

Introducing the short-hand notations

$$
\begin{aligned}
Q_{A} & =H_{R}, \\
Q_{A}^{\prime} & =\left(\frac{5}{9}+\frac{2}{3} \ln \frac{\alpha^{-2}}{2}\right) H_{R}^{\prime},
\end{aligned}
$$

we evaluate the second-order corrections as follows. After tracing out spins, we obtain for the $2^{3} S_{1}$ state

$$
\begin{aligned}
E\left(2^{3} S_{1}\right)_{\mathrm{SE}}^{\mathrm{sec}} & =\frac{1}{\pi}\left\{\left\langle 2^{3} S\left|Q_{A}^{\prime} \frac{1}{\left(E_{0}-H_{0}\right)^{\prime}} Q_{A}\right| 2^{3} S\right\rangle+\frac{2}{3}\left\langle 2^{3} S\left|Q_{B}^{\prime j} \frac{1}{\left(E_{0}-H_{0}\right)^{\prime}} Q_{B}^{j}\right| 2^{3} S\right\rangle\right. \\
& \left.+\frac{1}{3}\left\langle 2^{3} S\left|Q_{C}^{\prime j} \frac{1}{\left(E_{0}-H_{0}\right)^{\prime}} Q_{C}^{j}\right| 2^{3} S\right\rangle+\frac{1}{3}\left\langle 2^{3} S\left|Q_{D}^{\prime i j} \frac{1}{\left(E_{0}-H_{0}\right)^{\prime}} Q_{D}^{i j}\right| 2^{3} S\right\rangle\right\} .
\end{aligned}
$$

A similar result holds for the $2^{3} P$ centroid,

$$
\begin{aligned}
E\left(2^{3} P\right)_{\mathrm{SE}}^{\mathrm{sec}} & =\frac{1}{\pi}\left\{\left\langle 2^{3} P^{i}\left|Q_{A}^{\prime} \frac{1}{\left(E_{0}-H_{0}\right)^{\prime}} Q_{A}\right| 2^{3} P^{i}\right\rangle+\frac{2}{3}\left\langle 2^{3} P^{i}\left|Q_{B}^{\prime j} \frac{1}{\left(E_{0}-H_{0}\right)^{\prime}} Q_{B}^{j}\right| 2^{3} P^{i}\right\rangle\right. \\
& \left.+\frac{1}{3}\left\langle 2^{3} P^{i}\left|Q_{C}^{\prime j} \frac{1}{\left(E_{0}-H_{0}\right)^{\prime}} Q_{C}^{j}\right| 2^{3} P^{i}\right\rangle+\frac{1}{3}\left\langle 2^{3} P^{i}\left|Q_{D}^{\prime j k} \frac{1}{\left(E_{0}-H_{0}\right)^{\prime}} Q_{D}^{j k}\right| 2^{3} P^{i}\right\rangle\right\},
\end{aligned}
$$

where we assumed the normalization $\left\langle 2^{3} P^{i} \mid 2^{3} P^{i}\right\rangle=1$. This completes the description of the first term in Eq. (15) which is the electron self-energy contribution.

The second term in Eq. (15) is the one-loop vacuum polarization correction, for which we obtained [20]

$$
\begin{aligned}
E_{\mathrm{VP}}^{(7)}= & \frac{1}{\pi}\left\{\frac{8}{15} E_{0} E^{(4)}+\left(-\frac{E_{0}}{105}+\frac{137}{1050} Z^{2}-\frac{\pi^{2}}{54} Z^{2}-\frac{1}{15} Q_{7}-\frac{1}{15} Z^{2} \ln \alpha^{-2}\right) Z Q_{1}+\frac{1+13 Z}{105} Z Q_{3}-\frac{13}{210} Z Q_{4}\right. \\
& +\frac{13}{63} Q_{6 T}-\frac{4}{15} E^{(4)} Q_{7}-\frac{4}{15} E_{0} Z^{2} Q_{11}-\frac{8}{15} E_{0} Z^{2} Q_{12}+\frac{8}{15} E_{0} Z Q_{13}+\frac{8}{15} Z^{2} Q_{14}-\frac{8}{15} Z^{3} Q_{15}+\frac{4}{15} Z^{2} Q_{16} \\
& \left.-\frac{4}{15} Z Q_{17}-\frac{2}{15} Z^{2} Q_{21}-\frac{2}{15} Z^{2} Q_{22}-\frac{4}{15} Z Q_{24}+\frac{2}{15} Z Q_{28}-\frac{13}{210} Z Q_{51}-\frac{4}{15} E_{0}^{2} Z Q_{53}+\frac{Z^{2}}{15} Q_{57}\right\}+E_{\mathrm{VP}}^{\mathrm{sec}},
\end{aligned}
$$

with

Finally, the two-loop and three-loop radiative cor-

$$
E_{\mathrm{VP}}^{\mathrm{sec}}=-\frac{2}{15 \pi}\left\langle H_{R}^{\prime} \frac{1}{\left(E_{0}-H_{0}\right)^{\prime}} H_{R}\right\rangle .
$$


rections are obtained from the known hydrogenic results, keeping only the part proportional to the electronnucleus contact interaction, whereas the electron-electron contact interaction terms vanish because the nonrelativistic wave function is antisymmetric with respect to the exchange $\vec{r}_{1} \leftrightarrow \vec{r}_{2}$. Therefore, the two-loop correction is

$$
E_{\mathrm{rad} 2}^{(7)}=\frac{Z^{2}}{2 \pi^{2}} Q_{1} B_{50}
$$

where the coefficient $B_{50}$ is known only numerically, $B_{50}=-21.55447$ (13) [25]. Similarly, the three-loop radiative correction is given by [25]

$$
\begin{gathered}
E_{\mathrm{rad} 3}^{(7)}=\frac{Z}{2 \pi^{3}} Q_{1}\left[-\frac{568 a_{4}}{9}+\frac{85 \zeta(5)}{24}-\frac{121 \pi^{2} \zeta(3)}{72}\right. \\
-\frac{84071 \zeta(3)}{2304}-\frac{71 \ln ^{4} 2}{27}-\frac{239 \pi^{2} \ln ^{2} 2}{135} \\
\left.+\frac{4787 \pi^{2} \ln 2}{108}+\frac{1591 \pi^{4}}{3240}-\frac{252251 \pi^{2}}{9720}+\frac{679441}{93312}\right]
\end{gathered}
$$

where $a_{4}=\sum_{n=1}^{\infty} 1 /\left(2^{n} n^{4}\right)=0.517479061 \ldots$ This completes our evaluation of the $\alpha^{7} \mathrm{~m}$ contribution.

\section{ESTIMATION OF $\alpha^{8} m$ EFFECTS}

For the estimation of the radiative $\alpha^{8} m$ effects in helium, we employ the known hydrogenic results and pretend that they are proportional to the electron-nucleus contact interaction. Specifically, we use the results for the hydrogenic $2 s$ state of $\mathrm{He}^{+}[25]$

$$
\begin{aligned}
E_{\mathrm{rad} 1}^{(8+)}(\mathrm{hydr})= & \frac{Z^{7}}{8 \pi}\left(81.934_{\mathrm{SE}}+1.890 \mathrm{VP}\right) \\
E_{\mathrm{rad} 2}^{(8+)}(\mathrm{hydr})= & \frac{Z^{6}}{8 \pi^{2}}\left(-\frac{8}{27} \ln ^{3}\left[(Z \alpha)^{-2}\right]+0.639 \ln ^{2}\left[(Z \alpha)^{-2}\right]\right. \\
& \left.+41.387 \ln \left[(Z \alpha)^{-2}\right]-81.1 \pm 10\right)
\end{aligned}
$$

where the subscripts "SE" and "VP" denote the selfenergy and vacuum-polarization contributions, respectively. The three-loop contribution is small [26] and thus is neglected. The approximate $\alpha^{8} \mathrm{~m}$ corrections to the ionization energies of the $2^{3} S$ and $2^{3} P$ states of helium are obtained from the corresponding hydrogenic $2 s$ contributions by

$$
E^{(8+)}=E^{(8+)}(\text { hydr }) \frac{\left\langle\delta^{3}\left(r_{1}\right)+\delta^{3}\left(r_{2}\right)\right\rangle-Z^{3} / \pi}{Z^{3} / 8 \pi} .
$$

Specifically, we get contributions of $0.158(52) \mathrm{MHz}$ and $-0.048(16) \mathrm{MHz}$ for the ionization energies of the $2^{3} \mathrm{~S}$ and $2^{3} P$ states, correspondingly. We estimated the uncertainties to be $1 / 3$ of the corresponding numerical values; this estimate can be improved further once the $\alpha^{7} m$ contribution is verified.

\section{FINITE NUCLEAR SIZE EFFECT}

The last significant correction is due to the finite nuclear size, namely (in relativistic units)

$$
E_{\mathrm{fns}}=\frac{2 \pi}{3} Z \alpha\left\langle\sum_{a} \delta^{(3)}\left(r_{a}\right)\right\rangle R^{2}\left[1-(Z \alpha)^{2} \ln (m R Z \alpha)\right],
$$

where $R$ is the root-mean-square nuclear charge radius, and the expectation value of the Dirac $\delta$ functions is assumed to include the finite nuclear mass effects.

We note that Eq. (34) includes relativistic effects in the form of the leading logarithmic correction. Higher-order corrections to Eq. (34) were investigated for hydrogenlike atoms in Ref. [27]. Crude scaling shows that for helium they are negligible at the current level of precision and thus are neglected.

\section{NUMERICAL METHOD}

The spatial part of the helium wave function is expanded in a basis set of exponential functions of the form $[28,29]$

$$
\begin{aligned}
& \phi_{i}\left(r_{1}, r_{2}, r\right)=e^{-\alpha_{i} r_{1}-\beta_{i} r_{2}-\delta_{i} r} \pm\left(r_{1} \leftrightarrow r_{2}\right), \\
& \vec{\phi}_{i}\left(r_{1}, r_{2}, r\right)=\vec{r}_{1} e^{-\alpha_{i} r_{1}-\beta_{i} r_{2}-\delta_{i} r} \pm\left(r_{1} \leftrightarrow r_{2}\right)
\end{aligned}
$$

for the $S$ and $P$ states, correspondingly. The calculation of matrix elements of the nonrelativistic Hamiltonian is performed with help of the formula

$\frac{1}{16 \pi^{2}} \int d^{3} r_{1} \int d^{3} r_{2} \frac{e^{-\alpha r_{1}-\beta r_{2}-\delta r}}{r_{1} r_{2} r}=\frac{1}{(\alpha+\beta)(\beta+\delta)(\delta+\alpha)}$.

The results for integrals with any additional powers of $r$ in the numerator can be obtained by differentiation with respect to the corresponding parameter $\alpha, \beta$, or $\delta$.

Matrix elements of relativistic corrections involve integrals with additional inverse powers of $r_{1}, r_{2}$, and $r$. Formulas for such integrals can be obtained by integrating Eq. (37) with respect to the corresponding nonlinear parameter. This leads to the appearance of logarithmic and dilogarithmic functions; specifically,

$$
\frac{1}{16 \pi^{2}} \int d^{3} r_{1} \int d^{3} r_{2} \frac{e^{-\alpha r_{1}-\beta r_{2}-\delta r}}{r_{1} r_{2} r^{2}}=\frac{1}{(\alpha+\beta)(\alpha-\beta)} \ln \left(\frac{\alpha+\delta}{\beta+\delta}\right)
$$


TABLE II. Second-order corrections for the $2{ }^{3} S$ state; the prime on the sum means exclusion of the reference state.

\begin{tabular}{|c|c|c|}
\hline & $\begin{array}{c}\text { Intermediate } \\
\text { state }\end{array}$ & $2{ }^{3} S$ \\
\hline$\sum_{n}^{\prime} \frac{1}{E_{0}-E_{n}}\left\langle{ }^{3} S\left|H_{R}^{\prime}\right| n^{3} S\right\rangle\left\langle n^{3} S\left|H_{R}\right|^{3} S\right\rangle$ & ${ }^{3} S$ & 203.050945 \\
\hline $2 / 3 \pi \sum_{n} \frac{1}{E_{0}-E_{n}}\left\langle{ }^{3} S\left|Q_{B}^{\prime i}\right| n^{3} P^{i}\right\rangle\left\langle n^{3} P^{j}\left|Q_{B}^{j}\right|^{3} S\right\rangle$ & ${ }^{3} P^{e}$ & -0.003868 \\
\hline $1 / 3 \pi \sum_{n} \frac{1}{E_{0}-E_{n}}\left\langle n^{3} D^{i j}\left|Q_{D}^{i j}\right|^{3} S\right\rangle^{2}$ & ${ }^{3} D^{e}$ & -0.001225 \\
\hline
\end{tabular}

TABLE III. Second-order corrections for the $2{ }^{3} P$ state (centroid). Normalization is according to $\left\langle P^{i} \mid P^{i}\right\rangle=\left\langle D^{i j} \mid D^{i j}\right\rangle=$ $\left\langle F^{i j k} \mid F^{i j k}\right\rangle=1$.

\begin{tabular}{lcc}
\hline \hline & $\begin{array}{c}\text { Intermediate } \\
\text { state }\end{array}$ & $2^{3} P$ \\
\hline$\sum_{n}^{\prime} \frac{1}{E_{0}-E_{n}}\left\langle{ }^{3} P^{i}\left|H_{R}^{\prime}\right| n^{3} P^{i}\right\rangle\left\langle n^{3} P^{k}\left|H_{R}\right|^{3} P^{k}\right\rangle$ & ${ }^{3} P^{o}$ & 190.798218 (3) \\
$\sum_{n}^{\prime} \frac{1}{E_{0}-E_{n}}\left\langle{ }^{3} P^{i}\left|H_{\mathrm{exch}}^{(5)}\right| n^{3} P^{i}\right\rangle\left\langle n^{3} P^{k}\left|H_{R}\right|^{3} P^{k}\right\rangle$ & ${ }^{3} P^{o}$ & $0.000059(2)$ \\
$1 / 3 \pi \sum_{n}^{\prime} \frac{1}{E_{0}-E_{n}}\left\langle{ }^{3} P^{i}\left|i \epsilon^{i j k} Q_{B}^{\prime j}\right| n^{3} P^{k}\right\rangle\left\langle n^{3} P^{l}\left|i \epsilon^{l m n} Q_{B}^{m}\right|^{3} P^{n}\right\rangle$ & ${ }^{3} P^{o}$ & -0.008025 \\
$2 / 3 \pi \sum_{n} \frac{1}{E_{0}-E_{n}}\left\langle{ }^{3} P^{i}\left|Q_{B}^{\prime j}\right| n^{3} D^{i j}\right\rangle\left\langle n^{3} D^{l m}\left|Q_{B}^{l}\right|^{3} P^{m}\right\rangle$ & ${ }^{3} D^{o}$ & -0.000555 \\
$1 / 6 \pi \sum_{n} \frac{1}{E_{0}-E_{n}}\left\langle{ }^{3} P^{i}\left|i \epsilon^{i j k} Q_{C}^{\prime j}\right| n^{1} P^{k}\right\rangle\left\langle n^{1} P^{l}\left|i \epsilon^{l m n} Q_{C}^{m}\right|^{3} P^{n}\right\rangle$ & ${ }^{1} P^{o}$ & -0.028515 \\
$1 / 3 \pi \sum_{n} \frac{1}{E_{0}-E_{n}}\left\langle{ }^{3} P^{i}\left|Q_{C}^{\prime j}\right| n^{1} D^{i j}\right\rangle\left\langle n^{1} D^{l m}\left|Q_{C}^{l}\right|^{3} P^{m}\right\rangle$ & ${ }^{1} D^{o}$ & -0.000106 \\
$1 / 5 \pi \sum_{n}^{\prime} \frac{1}{E_{0}-E_{n}}\left\langle n^{3} P^{i}\left|Q_{D}^{i k}\right|^{3} P^{k}\right\rangle^{2}$ & ${ }^{3} P^{o}$ & -0.002429 \\
$2 / 9 \pi \sum_{n} \frac{1}{E_{0}-E_{n}}\left\langle n^{3} D^{i j}\left|i \epsilon^{i k l} Q_{D}^{j k}\right|^{3} P^{l}\right\rangle$ & -0.000015 \\
$1 / 3 \pi \sum_{n} \frac{1}{E_{0}-E_{n}}\left\langle n^{3} F^{i j k}\left|Q_{D}^{i j}\right|^{3} P^{k}\right\rangle^{2}$ & ${ }^{3} D^{o}$ & -0.000495 \\
\hline \hline
\end{tabular}

$$
\frac{1}{16 \pi^{2}} \int d^{3} r_{1} \int d^{3} r_{2} \frac{e^{-\alpha r_{1}-\beta r_{2}-\delta r}}{r_{1}^{2} r_{2} r^{2}}=\frac{1}{2 \beta}\left[\frac{\pi^{2}}{6}+\frac{1}{2} \ln ^{2}\left(\frac{\alpha+\beta}{\beta+\delta}\right)+\operatorname{Li}_{2}\left(1-\frac{\alpha+\delta}{\alpha+\beta}\right)+\operatorname{Li}_{2}\left(1-\frac{\alpha+\delta}{\beta+\delta}\right)\right] .
$$

Other integrals for relativistic corrections are obtained by differentiating the two basic formulas above.

In our calculation of the $\alpha^{7} m$ contribution, we encounter operators involving $\ln r+\gamma$, where $\gamma$ stands for the Euler's gamma constant. For the evaluation of these operators we obtained the following formulas

$$
\frac{1}{16 \pi^{2}} \int d^{3} r_{1} \int d^{3} r_{2} e^{-\alpha r_{1}-\beta r_{2}-\delta r} 4 \pi \delta\left(r_{1}\right) \frac{(\ln r+\gamma)}{r}=\frac{1-\ln (\beta+\delta)}{(\beta+\delta)^{2}},
$$

and

$$
\begin{gathered}
\frac{1}{16 \pi^{2}} \int d^{3} r_{1} \int d^{3} r_{2} \frac{e^{-\alpha r_{1}-\beta r_{2}-\delta r}}{r_{1} r_{2} r}(\ln r+\gamma)=\frac{1}{(\alpha-\beta)(\alpha+\beta)}\left[\frac{\ln (\alpha+\delta)}{\alpha+\delta}-\frac{\ln (\beta+\delta)}{\beta+\delta}\right], \\
\frac{1}{16 \pi^{2}} \int d^{3} r_{1} \int d^{3} r_{2} \frac{e^{-\alpha r_{1}-\beta r_{2}-\delta r}}{r_{1} r_{2} r^{2}}(\ln r+\gamma)=\frac{1}{2(\alpha-\beta)(\alpha+\beta)}\left[\ln ^{2}(\beta+\delta)-\ln ^{2}(\alpha+\delta)\right], \\
\frac{1}{16 \pi^{2}} \int d^{3} r_{1} \int d^{3} r_{2} \frac{e^{-\alpha r_{1}-\beta r_{2}-\delta r}}{r_{1}^{2} r_{2} r^{2}}(\ln r+\gamma)=\frac{1}{2 \beta}\left\{\frac{1}{2} \ln \left(\frac{\alpha-\beta}{\alpha+\beta}\right)\left[\ln ^{2}(\alpha+\delta)-\ln ^{2}(\beta+\delta)\right]\right. \\
\left.+\ln (\alpha+\delta)\left[\operatorname{Li}_{2}\left(\frac{-\beta+\delta}{\alpha+\delta}\right)-\operatorname{Li}_{2}\left(\frac{\beta+\delta}{\alpha+\delta}\right)\right]+\operatorname{Li}_{3}\left(\frac{-\beta+\delta}{\alpha+\delta}\right)-\operatorname{Li}_{3}\left(\frac{\beta+\delta}{\alpha+\delta}\right)\right\},
\end{gathered}
$$

where the last formula is valid for $\alpha>\beta$. The result for $\alpha<\beta$ is obtained by an analytic continuation with help 
of the identities

$$
\begin{aligned}
\operatorname{Li}_{2}(-z)+\mathrm{Li}_{2}\left(-z^{-1}\right) & =-\frac{\pi^{2}}{6}-\frac{\ln ^{2}(z)}{2} \\
\mathrm{Li}_{3}(-z)-\mathrm{Li}_{3}\left(-z^{-1}\right) & =-\frac{\pi^{2}}{6} \ln (z)-\frac{1}{6} \ln ^{3}(z) .
\end{aligned}
$$

In our calculation, we have derived explicit formulas for the expectation values of all $Q_{i}$ operators, and they involve the combination of the above expressions with the additional rational function of $\alpha, \beta$, and $\delta$.

\section{RESULTS}

Table I presents our numerical results for the relativistic corrections to the Bethe logarithm, obtained previously in Ref. [18]. Numerical values of the second-order corrections are summarized in Table II for the $2^{3} S$ state and in Table III for the $2^{3} P$ centroid. The uncertainties present for some of the matrix elements are negligible at the level of uncalculated higher-order contributions. Expectation values of various first-order operators are listed in Table IV. The matrix elements $Q_{i}$ with $i \leq 50$ have already been evaluated in our previous investigations (see Tables I and II of Ref. [22]), whereas the operators with $i>50$ are first encountered in the present work. The numerical uncertainties for $Q_{i}$ 's are smaller than the last digit shown.

Table V summarizes our calculation of the $\alpha^{7} \mathrm{~m}$ contributions to the energies of the $2^{3} S$ and $2^{3} P$ states of helium. In order to obtain contributions to the ionization energy, we need to subtract the corresponding corrections for the $1 S$ state of the $\mathrm{He}^{+}$ion, listed in the last column of the table. The hydrogenic formulas for $E_{\mathrm{SE}}^{(7)}\left(\mathrm{He}^{+}\right)$and $E_{\mathrm{L}}^{(7)}\left(\mathrm{He}^{+}\right)$are obtained from Refs. [30, 31] as follows

$$
\begin{aligned}
E_{\mathrm{SE}}^{(7)}\left(\mathrm{He}^{+}, 1 S\right)= & \frac{Z^{6}}{\pi}\left\{-\frac{121}{60}+\frac{5}{2} \zeta(3)-\frac{5}{18} \pi^{2}-\frac{61}{90} \ln 2\right. \\
& -3 \ln ^{2} 2+\ln (Z \alpha)\left[\frac{163}{30}-4 \ln (2 \Lambda)\right] \\
& \left.-\frac{5}{3} \ln \Lambda-\frac{22}{3} \ln 2 \ln \Lambda+\ln ^{2} \Lambda\right\}, \\
E_{\mathrm{L}}^{(7)}\left(\mathrm{He}^{+}, 1 S\right)= & \frac{Z^{6}}{\pi}\left\{\beta+\left(\frac{5}{3}+\frac{22}{3} \ln 2\right) \ln \left[\frac{\Lambda}{(Z \alpha)^{2}}\right]\right. \\
& \left.-\ln ^{2}\left[\frac{\Lambda}{(Z \alpha)^{2}}\right]\right\},
\end{aligned}
$$

where $\beta=\beta_{1}+\beta_{2}+\beta_{3}=27.25990948$ and $Z=2$. The sum $E_{\mathrm{SE}}^{(7)}\left(\mathrm{He}^{+}\right)+E_{\mathrm{L}}^{(7)}\left(\mathrm{He}^{+}\right)$does not depend on the cutoff parameter $\Lambda$. In order to be consistent with our present calculations for atomic He, one should set the cutoff parameter as $\Lambda \rightarrow \alpha^{2}$.

We note a strong cancellation between the $\mathrm{He}$ and $\mathrm{He}^{+}$corrections, which reflects the fact that the dominant contribution to the $2^{3} S$ and $2^{3} P$ energies comes from the $1 s$ electron. The resulting $\alpha^{7} m$ correction to the ionization energy is in agreement with our previous approximate predictions [17] based on the known $\mathrm{He}^{+}$ Lamb shift.

Table VI summarizes all known theoretical contributions to the ionization energies of the $2^{3} S$ and $2^{3} P$ states of helium. The contributions up to order $\alpha^{6} \mathrm{~m}$ correspond to those from our review [17], with the updated value of the Rydberg constant [33]. The finite nuclear size correction is calculated with the charge radius obtained from the recent measurement of the muonic helium Lamb shift [15]. We find that the effects of order $\alpha^{7} m$ and $\alpha^{8} m$ shift the $2^{3} S-2^{3} P$ transition frequency by $-8.447 \mathrm{MHz}$ and $0.206(54) \mathrm{MHz}$, respectively.

Table VII compares our final theoretical predictions with experimental results. There are three accurately measured transitions in He that involve the $2^{3} S$ and $2^{3} P$ states. The theoretical transition energy $E\left(2^{3} S-2^{3} P\right)_{\text {theo }}=276736495.620(54) \mathrm{MHz}$ is in very good agreement with the experimental result $E\left(2^{3} S-2^{3} P\right)_{\exp }=276736495.6000(14) \mathrm{MHz}$ from Ref. [32], while for the other two transitions, $2^{3} S-3^{3} D_{1}$ and $2^{3} P_{0}-3^{3} D_{1}$, theory and experiment disagree by about $0.5 \mathrm{MHz}$.

\section{DISCUSSION}

The theoretical energies contain the nuclear charge radius $R$ as a parameter, through the finite nuclear size correction given by Eq. (34). By comparing the theoretical predictions with high-precision experimental results (particularly, the $2^{3} S-2^{3} P$ transition energy [32]), one can determine $R$. The present theoretical accuracy is in principle sufficient for a determination of the nuclear radius with an accuracy of about $1 \%$. However, the unexplained discrepancy between theory and experiment for the $2^{3} S-3^{3} D$ and $2^{3} P-3^{3} D$ transitions does not allow us to do this.

Disagreements between theory and experiment for transitions involving $D$ states have already been reported previously $[16,17,37]$. The present calculation reduces the discrepancy for triplet states from $1 \mathrm{MHz}$ to $0.5 \mathrm{MHz}$. However, the theoretical uncertainty due to uncalculated higher-order effects is now reduced by an order of magnitude, so the relative discrepancy with experiment increased drastically, reaching $15 \sigma$ for the $2^{3} P_{0}-3^{3} D_{1}$ transition.

Bearing in mind the two different measurements, both of which show similar deviations from theory, we conclude that the most plausible explanation of the discrepancy would be some unknown theoretical contribution shifting the $2^{3} S$ and $2^{3} P$ states by approximately the same value. For this reason we postpone the determination of the $\alpha$-particle charge radius by means of the atomic spectroscopy until this unknown correction or a mistake in our calculations is identified. 


\section{ACKNOWLEDGMENTS}

K.P. and V.P. acknowledge support from the National Science Center (Poland) Grant No.
2017/27/B/ST2/02459. V.A.Y. acknowledges support from the Russian Science Foundation (Grant No. 20-62-46006). V.P. acknowledges additional support from the Czech Science Foundation - GAČR (Grant No. P209/18-00918S)
[1] E. Tiesinga, P. J. Mohr, D. B. Newell, and B. N. Taylor, Rev. Mod. Phys. in print (2021).

[2] S. Alighanbari et al., Nature 581, 152 (2020).

[3] S. Patra et al., Science 369, 1238 (2020).

[4] Z.-T. Lu, P. Mueller, G. W. F. Drake, W. Nörtershäuser, S. C. Pieper, and Z.-C. Yan, Rev. Mod. Phys. 85, 1383 (2013).

[5] B. Maaß, T. Hüther, K. König, J. Krämer, J. Krause, A. Lovato, P. Müller, K. Pachucki, M. Puchalski, R. Roth, R. Sánchez, F. Sommer, R. B. Wiringa, and W. Nörtershäuser, Phys. Rev. Lett. 122, 182501 (2019).

[6] K. Pachucki and V. A. Yerokhin, J. Phys. Chem. Ref. Data 44, 031206 (2015).

[7] K. Blaum et al., Phys. Script. T152, 014017 (2013).

[8] A. Beyer, L. Maisenbacher, A. Matveev, R. Pohl, K. Khabarova, A. Grinin, T. Lamour, D. C. Yost, T. W. Hänsch, N. Kolachevsky, and T. Udem, Science 358, 79 (2017).

[9] N. Bezginov, T. Valdez, M. Horbatsch, A. Marsman, A. C. Vutha, and E. A. Hessels, Science 365, 1007 (2019).

[10] H. Fleurbaey et al., Phys. Rev. Lett. 120, 183001 (2018).

[11] A. Grinin et al., Science 370, 1061 (2020).

[12] R. Pohl et al., Nature (London) 466, 213 (2010).

[13] A. Antognini et al., Science 339, 417 (2013).

[14] J.-P. Karr, D. Marchand, and E. Voutier, Nat. Rev. Phys. 2, 601 (2020).

[15] J. J. Krauth et al., Nature 589, 527 (2021).

[16] A. Wienczek, K. Pachucki, M. Puchalski, V. Patkóš, and V. A. Yerokhin, Phys. Rev. A 99, 052505 (2019).

[17] K. Pachucki, V. Patkóš, and V. A. Yerokhin, Phys. Rev. A 95, 062510 (2017).

[18] V. A. Yerokhin, V. Patkóš, and K. Pachucki, Phys. Rev. A 98, 032503 (2018), ibid. 103, 029901(E) (2021).

[19] V. Patkóš, V. A. Yerokhin, and K. Pachucki, Phys. Rev. A 101, 062516 (2020), ibid. 103, 029902(E) (2021).
[20] Patkóš, V. A. Yerokhin, and K. Pachucki, Phys. Rev. A 103, 012803 (2021).

[21] K. Pachucki, Phys. Rev. A 74, 022512 (2006).

[22] V. Patkóš, V. A. Yerokhin, and K. Pachucki, Phys. Rev. A 94, 052508 (2016).

[23] V. Patkóš, V. A. Yerokhin, and K. Pachucki, Phys. Rev. A 95, 012508 (2017).

[24] K. Pachucki and V. A. Yerokhin, Phys. Rev. A 79, 062516 (2009), [ibid. 80, 019902(E) (2009); ibid. 81, 039903(E) (2010)].

[25] V. A. Yerokhin, K. Pachucki, and V. Patkóš, Ann. Phys. (Leipzig) 531, 1800324 (2019).

[26] S. G. Karshenboim and V. A. Shelyuto, Phys. Rev. A 100, 032513 (2019).

[27] K. Pachucki, V. Patkóš, and V. A. Yerokhin, Phys. Rev. A 97, 062511 (2018).

[28] V. I. Korobov, Phys. Rev. A 61, 064503 (2000).

[29] V. I. Korobov, Phys. Rev. A 66, 024501 (2002).

[30] K. Pachucki, Ann. Phys. (NY) 226, 1 (1993).

[31] U. D. Jentschura, A. Czarnecki, and K. Pachucki, Phys. Rev. A 72, 062102 (2005).

[32] X. Zheng, Y. R. Sun, J.-J. Chen, W. Jiang, K. Pachucki, and S.-M. Hu, Phys. Rev. Lett. 119, 263002 (2017).

[33] CODATA internationally recommended values of the fundamental physical constants, physics.nist.gov/cuu/Constants/index.html, 2021.

[34] L. Morel, Z. Yao, P. Cladé, and S. Guellati-Khélifa, Nature 588, 61 (2020).

[35] C. Dorrer, F. Nez, B. de Beauvoir, L. Julien, and F. Biraben, Phys. Rev. Lett. 78, 3658 (1997).

[36] P.-L. Luo, J.-L. Peng, J. Hu, Y. Feng, L.-B. Wang, and J.-T. Shy, Phys. Rev. A 94, 062507 (2016).

[37] V. A. Yerokhin, V. Patkóš, M. Puchalski, and K. Pachucki, Phys. Rev. A 102, 012807 (2020).

[38] K. Pachucki and V. A. Yerokhin, J. Phys. Conf. Ser. 264, 012007 (2011). 
TABLE IV. Expectation values of various operators for the $2^{3} S$ and $2^{3} P$ states, $\vec{P}=\vec{p}_{1}+\vec{p}_{2}, \vec{p}=1 / 2\left(\vec{p}_{1}-\vec{p}_{2}\right)$.

\begin{tabular}{|c|c|c|c|}
\hline & & $2^{3} S$ & $2^{3} P$ \\
\hline$\overline{Q_{1}}$ & $4 \pi \delta^{3}\left(r_{1}\right)$ & 16.592071 & 15.819309 \\
\hline$Q_{2}$ & $4 \pi \delta^{3}(r)$ & 0 & 0 \\
\hline$Q_{3}$ & $4 \pi \delta^{3}\left(r_{1}\right) / r_{2}$ & 4.648724 & 4.349766 \\
\hline$Q_{4}$ & $4 \pi \delta^{3}\left(r_{1}\right) p_{2}^{2}$ & 2.095714 & 4.792830 \\
\hline$Q_{5}$ & $4 \pi \delta^{3}(r) / r_{1}$ & 0 & 0 \\
\hline$Q_{6 T}$ & $4 \pi \vec{p} \delta^{3}(r) \vec{p}$ & 0.028099 & 0.077524 \\
\hline$Q_{7}$ & $1 / r$ & 0.268198 & 0.266641 \\
\hline$Q_{8}$ & $1 / r^{2}$ & 0.088906 & 0.094057 \\
\hline$Q_{9}$ & $1 / r^{3}$ & 0.038861 & 0.047927 \\
\hline$Q_{10}$ & $1 / r^{4}$ & 0.026567 & 0.043348 \\
\hline$Q_{11}$ & $1 / r_{1}^{2}$ & 4.170446 & 4.014865 \\
\hline$Q_{12}$ & $1 /\left(r_{1} r_{2}\right)$ & 0.560730 & 0.550342 \\
\hline$Q_{13}$ & $1 /\left(r_{1} r\right)$ & 0.322696 & 0.317639 \\
\hline$Q_{14}$ & $1 /\left(r_{1} r_{2} r\right)$ & 0.186586 & 0.198346 \\
\hline$Q_{15}$ & $1 /\left(r_{1}^{2} r_{2}\right)$ & 1.242704 & 1.196631 \\
\hline$Q_{16}$ & $1 /\left(r_{1}^{2} r\right)$ & 1.164599 & 1.109463 \\
\hline$Q_{17}$ & $1 /\left(r_{1} r^{2}\right)$ & 0.112360 & 0.121112 \\
\hline$Q_{18}$ & $\left(\vec{r}_{1} \cdot \vec{r}\right) /\left(r_{1}^{3} r^{3}\right)$ & 0.011331 & 0.030284 \\
\hline$Q_{19}$ & $\left(\vec{r}_{1} \cdot \vec{r}\right) /\left(r_{1}^{3} r^{2}\right)$ & 0.054635 & 0.075373 \\
\hline$Q_{20}$ & $r_{1}^{i} r_{2}^{j}\left(r^{i} r^{j}-3 \delta^{i j} r^{2}\right) /\left(r_{1}^{3} r_{2}^{3} r\right)$ & 0.027082 & 0.090381 \\
\hline$Q_{21}$ & $p_{2}^{2} / r_{1}^{2}$ & 0.751913 & 1.410228 \\
\hline$Q_{22}$ & $\vec{p}_{1} / r_{1}^{2} \vec{p}_{1}$ & 16.720479 & 15.925672 \\
\hline$Q_{23}$ & $\vec{p}_{1} / r^{2} \vec{p}_{1}$ & 0.243754 & 0.279229 \\
\hline$Q_{24}$ & $p_{1}^{i}\left(r^{i} r^{j}+\delta^{i j} r^{2}\right) /\left(r_{1} r^{3}\right) p_{2}^{j}$ & 0.002750 & -0.097364 \\
\hline$Q_{25}$ & $P^{i}\left(3 r^{i} r^{j}-\delta^{i j} r^{2}\right) / r^{5} P^{j}$ & 0.062031 & -0.060473 \\
\hline$Q_{26}$ & $p_{2}^{k} r_{1}^{i} / r_{1}^{3}\left(\delta^{j k} r^{i} / r-\delta^{i k} r^{j} / r-\delta^{i j} r^{k} / r-r^{i} r^{j} r^{k} / r^{3}\right) p_{2}^{j}$ & -0.009102 & 0.071600 \\
\hline$Q_{27}$ & $p_{1}^{2} p_{2}^{2}$ & 0.488198 & 1.198492 \\
\hline$Q_{28}$ & $p_{1}^{2} / r_{1} p_{2}^{2}$ & 1.597727 & 3.883405 \\
\hline$Q_{29}^{28}$ & $\vec{p}_{1} \times \vec{p}_{2} / r \vec{p}_{1} \times \vec{p}_{2}$ & 0.070535 & 0.399306 \\
\hline$Q_{30}$ & $p_{1}^{k} p_{2}^{l}\left(-\delta^{j l} r^{i} r^{k} / r^{3}-\delta^{i k} r^{j} r^{l} / r^{3}+3 r^{i} r^{j} r^{k} r^{l} / r^{5}\right) p_{1}^{i} p_{2}^{j}$ & -0.034780 & -0.187305 \\
\hline$Q_{31}$ & $4 \pi \delta^{3}\left(r_{1}\right) \vec{p}_{1} \cdot \vec{p}_{2}$ & 0.040294 & -0.457224 \\
\hline$Q_{32}$ & $\left(\vec{r}_{1} \cdot \vec{r}_{2}\right) /\left(r_{1}^{3} r_{2}^{3}\right)$ & -0.005797 & -0.032383 \\
\hline$Q_{33}$ & $\vec{p}_{1} \cdot \vec{p}_{2}$ & 0.007442 & -0.064572 \\
\hline$Q_{34}$ & $\vec{P} / r_{1} \vec{P}$ & 4.974707 & 4.730359 \\
\hline$Q_{35}$ & $\vec{P} / r \vec{P}$ & 1.232372 & 1.127146 \\
\hline$Q_{36}$ & $\vec{P} / r_{1}^{2} \vec{P}$ & 17.504835 & 16.972775 \\
\hline$Q_{37}$ & $\vec{P} /\left(r_{1} r_{2}\right) \vec{P}$ & 2.489592 & 2.291176 \\
\hline$Q_{38}$ & $\vec{P} /\left(r_{1} r\right) \vec{P}$ & 1.454007 & 1.350214 \\
\hline$Q_{39}$ & $\vec{P} / r^{2} \vec{P}$ & 0.438804 & 0.413144 \\
\hline$Q_{40}$ & $p_{1}^{2} p_{2}^{2} P^{2}$ & 10.324509 & 24.527699 \\
\hline$Q_{41}$ & $P^{2} p_{1}^{i}\left(r^{i} r^{j}+\delta^{i j} r^{2}\right) / r^{3} p_{2}^{j}$ & 0.151748 & 0.067201 \\
\hline$Q_{42}$ & $p_{1}^{i}\left(r_{1}^{i} r_{1}^{j}+\delta^{i j} r_{1}^{2}\right) / r_{1}^{4} P^{j}$ & 33.461709 & 31.489835 \\
\hline$Q_{43}$ & $p_{1}^{i}\left(r_{1}^{i} r_{1}^{j}+\delta^{i j} r_{1}^{2}\right) /\left(r_{1}^{3} r_{2}\right) P^{j}$ & 2.486269 & 2.217310 \\
\hline$Q_{44}$ & $p_{1}^{i} p_{2}^{k}\left(r_{1}^{i} r_{1}^{j}+\delta^{i j} r_{1}^{2}\right) / r_{1}^{3} p_{2}^{k} P^{j}$ & 1.100915 & 2.527505 \\
\hline$Q_{45}$ & $p_{2}^{i}\left(r^{i} r^{j}+\delta^{i j} r^{2}\right)\left(r_{1}^{j} r_{1}^{k}+\delta^{j k} r_{1}^{2}\right) /\left(r_{1}^{3} r^{3}\right) P^{k}$ & 0.540877 & 0.467623 \\
\hline$Q_{46}$ & $p_{1}^{i}\left(r_{1}^{i} r_{1}^{j}+\delta^{i j} r_{1}^{2}\right)\left(r_{2}^{j} r_{2}^{k}+\delta^{j k} r_{2}^{2}\right) /\left(r_{1}^{3} r_{2}^{3}\right) p_{2}^{k}$ & 0.006782 & -0.201826 \\
\hline$Q_{47}$ & $\left(\vec{r}_{1} \cdot \vec{r}_{2}\right) /\left(r_{1}^{3} r_{2}^{2}\right)$ & -0.008117 & -0.028621 \\
\hline$Q_{48}$ & $r_{1}^{i} r^{j}\left(r_{1}^{i} r_{1}^{j}-3 \delta^{i j} r_{1}^{2}\right) /\left(r_{1}^{4} r^{3}\right)$ & -0.036861 & -0.057404 \\
\hline$Q_{49}$ & $r_{1}^{i} r^{j}\left(r_{2}^{i} r_{2}^{j}-3 \delta^{i j} r_{2}^{2}\right) /\left(r_{1}^{3} r_{2} r^{3}\right)$ & -0.089086 & -0.126780 \\
\hline$Q_{50}$ & $p_{2}^{k} r_{1}^{i} / r_{1}^{3}\left(\delta^{j k} r_{2}^{i} / r_{2}-\delta^{i k} r_{2}^{j} / r_{2}-\delta^{i j} r_{2}^{k} / r_{2}-r_{2}^{i} r_{2}^{j} r_{2}^{k} / r_{2}^{3}\right) p_{2}^{j}$ & 0.005856 & -0.092036 \\
\hline$Q_{51}$ & $4 \pi \vec{p}_{1} \delta^{3}\left(r_{1}\right) \vec{p}_{1}$ & 0.009993 & 0.270964 \\
\hline$Q_{52}$ & $4 \pi \delta^{3}\left(r_{1}\right) / r_{2}\left(\ln r_{2}+\gamma\right)$ & 8.125982 & 7.514290 \\
\hline$Q_{53}$ & $1 / r_{1}$ & 1.154664 & 1.133242 \\
\hline$Q_{54}$ & $1 / r^{4}(\ln r+\gamma)$ & 0.015481 & 0.009473 \\
\hline$Q_{55}$ & $1 / r^{5}$ & 0.017580 & 0.027240 \\
\hline$Q_{56}$ & $1 / r_{1}^{3}$ & -23.022535 & -21.886142 \\
\hline$Q_{57}$ & $1 / r_{1}^{4}$ & 25.511837 & 24.525751 \\
\hline$Q_{58}$ & $\left(\vec{r}_{1} \cdot \vec{r}\right) /\left(r_{1}^{3} r^{3}\right)(\ln r+\gamma)$ & 0.026515 & 0.038795 \\
\hline$Q_{59}$ & $1 /\left(r_{1} r^{3}\right)$ & 0.051914 & 0.069729 \\
\hline$Q_{60}$ & $\vec{p} / r^{3} \vec{p}$ & 0.072885 & 0.093877 \\
\hline$Q_{61}$ & $\vec{P} / r^{3} \vec{P}$ & 0.211990 & 0.226284 \\
\hline$Q_{62}$ & $r^{i} r^{j}\left(\delta^{i j} r_{1}^{2}-3 r_{1}^{i} r_{1}^{j}\right) /\left(r_{1}^{5} r^{3}\right)$ & -0.017688 & -0.051696 \\
\hline$Q_{63}$ & $r^{i} r^{j}\left(\delta^{i j} r_{1}^{2}-3 r_{1}^{i} r_{1}^{j}\right) /\left(r_{1}^{5} r^{3}\right)(\ln r+\gamma)$ & -0.045609 & -0.045395 \\
\hline$Q_{64}$ & $p^{i}\left(\delta^{i j} r^{2}-3 r^{i} r^{j}\right) / r^{5} p^{j}$ & 0.002731 & 0.021530 \\
\hline
\end{tabular}


TABLE V. Numerical results for individual contributions to $E^{(7)}$ for the $2^{3} S$ and $2^{3} P$ (centroid) states of helium, in units of $\alpha^{7} m$ if not specified explicitly.

\begin{tabular}{lccr}
\hline \hline Term & \multicolumn{1}{c}{$2^{3} S$} & $2^{3} P$ & $\mathrm{He}^{+}(1 S)$ \\
\hline$E_{L}^{(7)}$ & $-804.306(5)$ & $-767.828(10)$ & -785.107 \\
$E_{\mathrm{SE}}^{(7)}$ & -379.061 & -359.257 & -367.554 \\
$E_{\mathrm{VP}}^{(7)}$ & -36.094 & -34.381 & -34.716 \\
$E_{\text {rad2 }}^{(7)}$ & -72.471 & -69.096 & -69.885 \\
$E_{\text {rad3 }}^{(7)}$ & 0.223 & 0.213 & 0.215 \\
$E_{\text {exch }}^{(7)}$ & -10.639 & -9.950 & 0.000 \\
$E^{(7)}$ & $-1302.348(5)$ & $-1240.301(10)$ & -1257.046 \\
$E^{(7)}[\mathrm{MHz}]$ & $-177.320(1)$ & $-168.872(1)$ & -171.152 \\
He $-\mathrm{He}{ }^{+}[\mathrm{MHz}]$ & $-6.168(1)$ & $2.280(1)$ & \\
Prev. theory $[17]$ & $-5.2(1.3)$ & $2.9(0.7)$ & \\
\hline \hline
\end{tabular}

TABLE VI. Breakdown of theoretical contributions to the ionization (centroid) energies of the $2^{3} S$ and $2^{3} P$ states of ${ }^{4} \mathrm{He}$, in $\mathrm{MHz} . \quad R_{\infty} c=3.2898419602508(64) \times 10^{15} \mathrm{~Hz}[33], M / m_{e}=7294.29954142(24)[33], 1 / \alpha=137.035999206(11)$ [34], $R=1.67824(83) \mathrm{fm}$ [15]. NS denotes the finite nuclear size correction; NP stands for the nuclear polarizability correction. The uncertainty of the theoretical $\alpha^{2}$ contribution comes from the Rydberg constant; the uncertainty of the finite nuclear size correction comes from the nuclear radius.

\begin{tabular}{|c|c|c|c|c|c|}
\hline & $(m / M)^{0}$ & $(m / M)^{1}$ & $(m / M)^{2}$ & $(m / M)^{3}$ & Sum \\
\hline$\alpha^{2}$ & $-1152953922.384(2)$ & 164775.354 & -30.620 & 0.006 & $-1152789177.644(2)$ \\
\hline$\alpha^{5}$ & 3999.431 & -0.800 & & & 3998.632 \\
\hline$\alpha^{6}$ & 65.235 & -0.030 & & & 65.205 \\
\hline$\alpha^{7}$ & $-6.168(1)$ & & & & $-6.168(1)$ \\
\hline NP & -0.001 & & & & -0.001 \\
\hline Total & & & & & $-1152842742.231(52)$ \\
\hline Theory 2017 [17] & & & & & $-1152842741.4(1.3)$ \\
\hline \multicolumn{6}{|l|}{$2^{3} P$ : } \\
\hline$\alpha^{2}$ & $-876178284.857(2)$ & 61871.895 & -25.840 & 0.006 & $-876116438.795(2)$ \\
\hline$\alpha^{8}$ & $-0.048(16)$ & & & & $-0.048(16)$ \\
\hline NS & $-0.799(1)$ & & & & $-0.799(1)$ \\
\hline NP & 0.000 & & & & 0.000 \\
\hline Total & & & & & $-876106246.611(16)$ \\
\hline Theory 2017 [17] & & & & & $-876106246.0(7)$ \\
\hline
\end{tabular}

TABLE VII. Comparison of experimental results for various transitions with theoretical predictions, in MHz.

\begin{tabular}{|c|c|c|c|c|}
\hline Transition & Theory & \multicolumn{2}{|c|}{ Experiment } & Difference \\
\hline $2^{3} S-3^{3} D_{1}$ & $786823849.540(52)^{a}$ & $786823850.002(56)$ & {$[35]$} & $-0.462(76)$ \\
\hline $2^{3} P_{0}-3^{3} D_{1}$ & $510059754.863(16)^{a, b}$ & $510059755.352(28)$ & {$[36]$} & $-0.489(32)$ \\
\hline $2^{3} P-2^{3} S$ & $276736495.620(54)$ & $276736495.6000(14)$ & {$[32]^{b}$} & $0.020(54)$ \\
\hline
\end{tabular}

${ }^{a}$ using theoretical energy $E\left(3^{3} D_{1}\right)=366018892.691$ (23) from Ref. [37],

${ }^{b}$ using theoretical results for the $2^{3} P$ fine structure from Ref. [38]. 